Bioscientia Medicina: Journal of Biomedicine \& Translational Research

Journal Homepage: www.bioscmed.com

\title{
Epilepsy as a Presenting Symptom of Covid-19 in a Tertiary Private Hospital in Northern Mexico
}

\section{Sofia Lucila Rodriguez Rivera ${ }^{1 *}$, José Antonio Infante Cantú ${ }^{1}$, Héctor R. Martínez ${ }^{2}$, Enrique Caro Osorio $^{3}$}

${ }^{1}$ Pediatric Neurologist, Zambrano Hellion, TecSalud Hospital. Tecnológico de Monterrey, Monterrey, Nuevo León, Mexico

2 Neurologist, Zambrano Hellion, TecSalud Hospital. Tecnológico de Monterrey, Monterrey, Nuevo León, Mexico

${ }^{3}$ Neurosurgery, Zambrano Hellion, TecSalud Hospital. Tecnológico de Monterrey, Monterrey, Nuevo León, Mexico

A R T I C L E I N F O

Keywords:

Seizures

Epilepsy

COVID-19

SARS-CoV-2

*Corresponding author:

Sofia Lucila Rodríguez Rivera

E-mail address:

sofiardz85@gmail.com

All authors have reviewed and approved the final version of the manuscript.

https://doi.org/10.37275/bsm.v6i3.462

\begin{abstract}
A B S T R A C T
Background. Various manifestations of COVID-19 have been described in patients, including neurological. Few studies describe seizures as a presenting symptom. This study was aimed to identify clinical characteristics, type of epilepsy, and electroencephalographic findings in patients with epilepsy as a presenting symptom of COVID-19 in a tertiary private hospital. Methods: Descriptive, retrospective, observational and cross-sectional study. Inclusion criteria were patients with epilepsy as a presenting symptom of COVID-19 confirmed with Polymerase Chain Reaction (PCR) for SARS-CoV2 by nasopharyngeal swab from March 2020-July 2021 in a tertiary private hospital. Study variables were age, gender, type of epilepsy, comorbidities, and electroencephalographic findings. It was classified into three groups: acute symptomatic seizures, the onset of epilepsy, and uncontrolled epilepsy. Information was captured in Excel and analyzed in SPSS. Results: Of 203,987 patients with a confirmed diagnosis of COVID-19 in Nuevo León until July 2021, 10 patients $(0.004 \%)$ were included with seizures. Two patients had acute symptomatic seizures $(20 \%)$, four patients had onset seizures $(40 \%)$ and four patients (40\%) had uncontrolled epilepsy with an average epilepsy evolution time of 15.75 years. Focal seizures were predominant in $63 \%$. Electroencephalogram was abnormal in $90 \% \quad(50 \%$ focal frontotemporal sharp waves, $20 \%$ encephalopathic, $20 \%$ generalized spike-wave). Two patients $(20 \%)$ had status epilepticus. Conclusion: This study is important to carry out early detection in suspects with a previous neurological history and to avoid the spread of the coronavirus.
\end{abstract}

\section{Introduction}

Since the beginning of the year 2020, the world has been experiencing a catastrophic pandemic of coronavirus disease (COVID-19) that is caused by SARS-CoV-2.1 COVID-19 has spread to millions of people around the world and has caused millions of deaths. Various manifestations have been described, including neurological. ${ }^{2}$ They are classified into Central Nervous System related manifestations including headache, dizziness, impaired consciousness, acute cerebrovascular disease, epilepsy, and Peripheral Nervous System related manifestations such as hyposmia/anosmia, hypogeusia/ageusia, muscle pain, and Guillain-Barre syndrome.

Few studies describe seizures as a presenting symptom in patients with COVID-19.3 However, patients with COVID-19 may have hypoxia, multiorgan 
failure, severe metabolic and electrolyte disarrangements ${ }^{4}$; therefore, it is possible to expect acute symptomatic seizures to occur in these patients. Furthermore, a growing body of evidence indicates that neurotropism is one common feature of coronavirus. The objective was to identify clinical characteristics, type of epilepsy, and electroencephalographic findings in patients with epilepsy as a presenting symptom of COVID-19 in a tertiary private hospital.

\section{Methods}

This was a descriptive, retrospective, observational and cross-sectional study. Inclusion criteria were patients with epilepsy as a presenting symptom of COVID-19 confirmed with Polymerase Chain Reaction (PCR) for SARS-CoV2 by nasopharyngeal swab from March 2020-July 2021 in a tertiary private hospital. Exclusion criteria were files with incomplete data. Data were obtained from clinical records. Study variables were age, gender, type of epilepsy, comorbidities, and electroencephalographic findings. It was classified into three groups: acute symptomatic seizures, the onset of epilepsy, and uncontrolled epilepsy. The EEG was performed in patients at Neurophysiology Department, with a duration of half an hour, a velocity of $3 \mathrm{~cm} / \mathrm{s}$, in vigil and physiological sleep. The electroencephalographic tracing was recorded as either normal or abnormal. The EEG was considered normal when the study had an adequate integration of the stages of sleep, with symmetry and synchrony, without abnormal graphoelements. The abnormal EEG (with epileptiform activity) were the tracings with a focal or generalized activity of the spikes, sharp waves, spikewave complexes, polyspikes, or wave polyspike. Information was captured in Excel. Tests (measures of central tendency, graphs) were applied in the SPSS program.

\section{Results}

Of 203,987 patients with a confirmed diagnosis of COVID-19 in Nuevo León until July 2021, 10 patients $(0.004 \%)$ were included with seizures. The mean age of the studied population is 22.72 years old, DE+_23.11 (40 days -57 years old). The male gender is predominant with $70 \%$. Three groups were found: acute symptomatic seizures, the onset of epilepsy, and uncontrolled epilepsy.

Two patients had acute symptomatic seizures (20\%), four patients had onset seizures (40\%) and four patients (40\%) had uncontrolled epilepsy with an average epilepsy evolution time of 15.75 years. Regarding the type of epilepsy, focal seizures were predominant in 63\%. The most used antiepileptics were valproate and levetiracetam. $70 \%$ of comorbidities were neurological including autism, cerebral palsy, and migraine. $20 \%$ of the patients had encephalitis.

Electroencephalogram was abnormal in 90\% (50\% focal frontotemporal sharp waves, $20 \%$ encephalopathic, 20\% generalized spike-wave). Two patients $(20 \%)$ had status epilepticus. Eight patients did not show symptoms of COVID-19 before the presentation (80\%), 100\% had a fever in the course, and in all cases, epileptic seizures were the reason for consultation. $80 \%$ of patients required antiepileptic after hospital discharge.

\section{Discussion}

A meta-analysis based on 39 studies and 68,362 patients with COVID-19 showed that about $21 \%$ had neurological symptoms. The most frequent were headaches (4.6\%) and cerebrovascular events (4\%). Seizures occurred in $0.7 \% .^{5}$ Acute symptomatic seizures, new-onset seizures, uncontrolled or newonset epilepsy (focal motor, generalized tonic-clonic), convulsive and non-convulsive status epilepticus, and febrile seizures have been reported.

New-onset seizures can occur in people with COVID-19 in a variety of ways: focal motor seizures, generalized tonic-clonic seizures, convulsive and nonconvulsive status epilepticus, and post-SARS-CoV2 autoimmune encephalitis associated with new-onset refractory status epilepticus (NORSE). Additionally, many patients with severe COVID-19 may have changes in mental status.

Seizures are an important manifestation that can complicate the course of the disease in any seriously ill patient. In these patients, isolated seizures can rapidly escalate to convulsive status epilepticus or non- 
convulsive status epilepticus that is associated with high morbidity and mortality. Several cases of refractory status epilepticus are described as part of COVID-19 infection. Two cases responding to intravenous immunoglobulin therapy, and one was associated with anti-NMDA encephalitis. 6, 7, 8

So far, there is no evidence that COVID-19 infection alone exacerbates seizures in patients with established epilepsy. However, a recent systematic review suggested that patients with pre-existing neurological disorders (including epilepsy) and COVID-19 may develop exacerbation of their neurological problems and also severe COVID-19.9

Interictal epileptiform discharges, slow background rhythm, and non-convulsive status epilepticus have been reported. In one series, only a small proportion of patients (8 of 64) with electroencephalogram demonstrated diffuse slowing consistent with encephalopathy. 10 Three studies showed that many of the patients with COVID-19 and encephalopathy with seizures had epileptiform discharges on their EEG.11-13

A study of two patients with COVID-19 and encephalopathy suggested a unique EEG pattern with continuous, asymmetric, monomorphic, biphasic, delta slow waves with greater amplitude over both frontal areas and with a periodic organization. ${ }^{14}$ At the moment, the studies that investigated the EEG in patients with COVID-19 did not find a consistent and specific neurophysiological pattern in critically ill patients.

\section{Conclusions}

In conclusion, hypoxia, fever, electrolyte or metabolic disorders, systemic diseases or direct or indirect neurotropic effect of the SARS-CoV2 virus could favor the appearance of seizures in patients with COVID-19. In our study, patients with seizures were found as a presenting symptom of COVID-19, without finding a characteristic clinic pattern or electroencephalographic, which is important to know to carry out early detection in suspects or with a previous neurological history and to avoid the spread of the coronavirus.

\section{References}

1. Rothan HA, Byrareddy SN. The epidemiology and pathogenesis of coronavirus disease (COVID-19) outbreak. $J$ Autoimmun. 2020; 109: 102433.

2. Mao L., Wang M., Chen S., He Q., Chang J., Hong C. Neurological manifestations of hospitalized patients with COVID-19 in Wuhan, China: a retrospective case series study. medRxiv. 2020.

3. Kadono Y, Nakamura Y, Ogawa Y, et al. A case of COVID-19 infection presenting with a seizure following severe brain edema. Seizure. 2020; 80: 53-55.

4. Narula N, Joseph R, Katyal N, et al. Seizure and COVID-19: Association and review of potential mechanism. Neurol Psychiatry Brain Res. 2020; 38: 49-53.

5. Cagnazzo F, Arquizan C, Derraz I et al. Neurological manifestations of patients infected with the SARS-CoV-2: a systematic review of the literature. J Neurol. 2020; 267: 1-10.

6. Dono F, Nucera B, Lanzone J et al. Status epilepticus and COVID-19: A systematic review. Epilepsy Behav 2021; 118: 107887.

7. Manganotti P, Furlanis G, Ajčević M et al. Intravenous immunoglobulin response in new-onset refractory status epilepticus (NORSE) COVID-19 adult patients. J Neurol 2021; 268: 1-5.

8. Monti G, Giovannini G, Marudi A et al. AntiNMDA receptor encephalitis presenting as new-onset refractory status epilepticus in COVID-19. Seizure 2020; 81: 18-20.

9. Kubota T, Kuroda N (2020) Exacerbation of neurological symptoms and COVID-19 severity in patients with preexisting neurological disorders and COVID-19: a systematic review. Clin Neurol Neurosurg: 106349. 
10. Helms J, Kremer S, Merdji H, et al. Neurologic features in severe SARS-CoV-2 infection. New Engl J Med. 2020.

11. Anand P, Al-Faraj A, Sader E, Dashkoff J, Abdennadher M, Murugesan $\mathrm{R}$ et al. Seizure as the presenting symptom of COVID-19: a retrospective case series. Epilepsy Behav. 2020; 112:10733.

12. Pilato MS, Urban A, Alkawadri R, Barot NV, Castellano JF, Rajasekaran V et al. EEG findings in coronavirus disease. $\mathrm{J}$ Clin Neurophysiol. 2020.

13. Galanopoulou AS, Ferastraoaru V, Correa DJ, Cherian K, Duberstein S, Gursky J, Hanumanthu R, Hung C, Molinero I, Khodakivska O, Legatt AD, Patel P, Rosengard J, Rubens E, Sugrue W, Yozawitz E, Mehler MF, Ballaban-Gil K, Haut SR, Moshé SL, Boro A. EEG findings in acutely ill patients investigated for SARSCoV-2/COVID-19: a small case series preliminary report. Epilepsia Open, 2020; 5: 314-324.

14. Vellieux G, Rouvel-Tallec A, Jaquet $P$, Grinea A, Sonneville R, d'Ortho MP. COVID19 associated encephalopathy: is there a specific EEG pattern? Clin Neurophysiol. 2020; 131: 1928-1930. 